\title{
Using Infrared Thermography to Visualize the Invisible: Investigating Heat Transfer
}

\author{
By A. Dexter*, L. Chiesa*, and C. Xie ${ }^{* *}$ \\ *Dept. of Mechanical Engineering, Tufts University, Medford, MA 02138, amy.dexter@tufts.edu \\ ${ }^{* *}$ Concord Consortium, Concord, MA 01742, qxie@concord.org
}

\begin{abstract}
This article reports the results of an early study of practical infrared imaging technology in the classroom. The IR camera has the potential to be a powerful scientific tool that can offer invaluable insight into obscure heat transfer phenomena by enabling students to "see" and analyze otherwise invisible thermal concepts. In this paper, the IR camera is used to directly investigate heat and temperature in real time and used to determine the advantages and limitations of currently used simulation software with the final goal of increasing students' understanding of heat transfer.
\end{abstract}

\section{Introduction}

What is temperature? In the current classroom environment answering this seemingly simple question can be extremely challenging. The words "temperature" and "heat" are known to very young children, ages 2-3 [1], and are used in numerous everyday, nonscientific contexts throughout a child's life. Beyond simply the words, students experience a seemingly infinite number of moments involving thermal physics, with common questions like 'Why does the doorknob feel cooler than the desk?' and 'Why are my cheeks so red after coming in from recess?' Students often engage in discussions on these topics, but might find it extremely challenging to understand and explain these abstract concepts with the tools to which they currently have access. Like many phenomena in the natural world, temperature is "unseen," which makes it difficult for students to engage with and make sense of ideas like heat and temperature.

The understanding of heat transfer is important as it provides a foundation for other science and engineering concepts. Until now students have relied on books, thermometers and teachers as resources for explaining these abstract and unintuitive concepts thermal energy transport mechanisms, which often leave students with more questions than answers. With the use of a simple to use, non-contact tool infrared (IR) camera, students can now "see" the mechanisms behind heat transfer.

Historically, IR cameras have been used for scientific, engineering and military purposes and have been too expensive for use in the classroom. In the past decade the cost of IR cameras has decreased tremendously, and if this trend continues, the cost of an IR camera will be only a few hundred dollars within the next ten years making them affordable for use in K-12 classrooms where concepts of heat and temperature are first introduced [2]. This portable and easy-to-use tool has the power to transform the way students learn about mechanisms that drive heat transfer. Students will now have the opportunity to "see" and further explore these invisible thermal energy concepts. Along with providing thermal visualizations of scientific phenomena, the IR camera has the potential to transform the learning process, opening students' minds and creating an excitement towards technology.

\section{IR in the Classroom}

Many technologies that have been introduced into the classroom have only provided incremental improvements. This is partly a result of technology being implemented before being fully understood. The IR camera is a tool that has the potential not only to improve the way students learn, but to completely transform the classroom experience. There are currently a number of transformative tools and technologies, such as iPads [3,4] and LEGO robots, that have completely changed the way content and skills are presented, prompting students to be scientists, investigating the world around them and trying to understand what they see. The goal of the current research efforts presented here is to understand the IR technology before it is introduced into the classroom in order to utilize all that this technology has to offer from the very beginning.

So how can students and teachers use an IR camera in a classroom? The first step is simply to look at everyday objects. For instance, figure 1 shows how fur and hair insulate a dog in a cold house. One can see the substantial heat loss around the eyes and mouth but excellent heat retention in the surrounding sections of the body. Another interesting aspect of this image is the clear identification that the coldest part of a dog's body is the nose. A dog's nose is cold due to evaporative cooling caused by the dog licking its nose and fluids secreted inside the nose used to regulate its body temperature. 


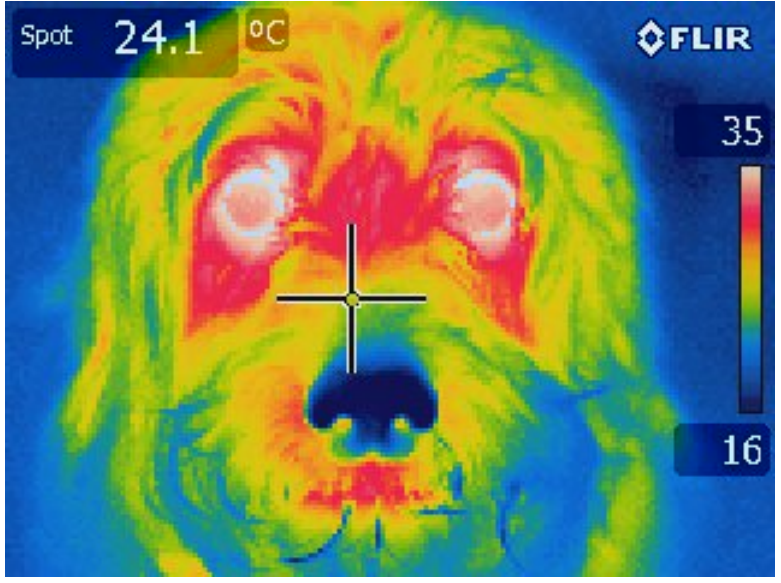

Figure 1. An IR portrait of a dogs face shows several concepts of heat transfer at work

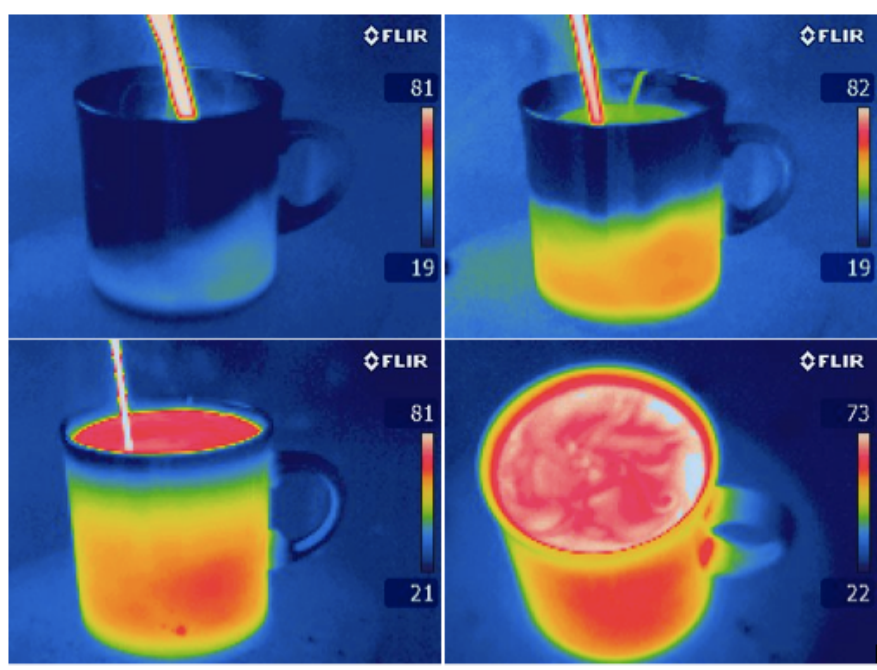

Figure 2. Series of screenshots from IR video showing thermal conduction between hot water and a coffee cup

The next step is to examine changes in an image with time. The series of images in figure 2 shows a very familiar process, the heating of a coffee cup by a hot liquid. Hot water is added to the coffee cup in the upper left image and continues until the cup is full in the lower left image. Both conduction and convection are illustrated in the figure shown above. As the hot liquid makes contact with the inner walls of the cup, thermal energy from the liquid is conducted to the outer wall of the cup. The cup and the liquid it contains will eventually cool as a result of losing thermal energy to the surrounding air via convection and to the table through conduction. The warmer liquid rises to the top and cools and as a result, increases in density, which causes the liquid to sink to the bottom where it begins to warm and the process starts over. The movement of the liquid in this process is captured in the last image. Common activities, similar to the one described, can lead to discussion about heat flux, temperature and material properties and encourage students to explore the effects these factors have on the outcome.

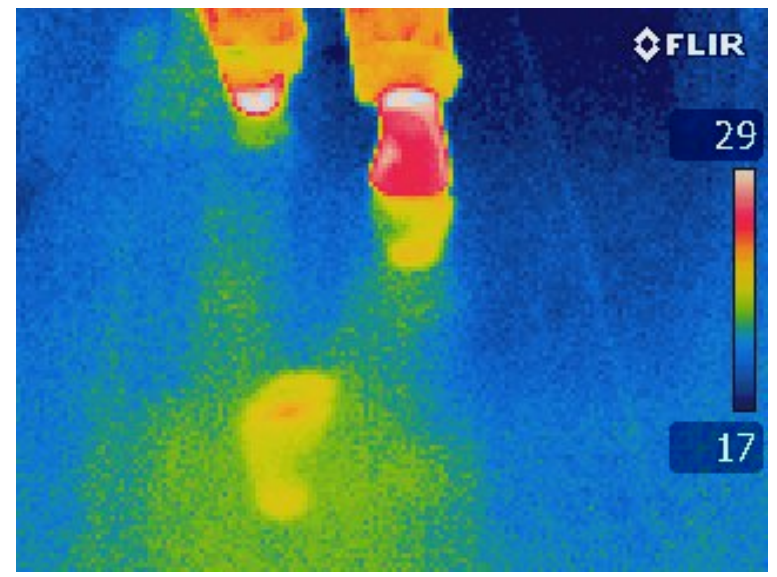

Figure 3. IR image showing thermal shadows of

footprints on a floor resulting from conduction from contact between the foot and floor

The third step is to investigate indirect effects such residual heat sometimes referred to as "thermal shadows" [5]. For example, based on residual heat, one can look at an empty couch and determine the number of people previously sitting on the couch and roughly how long they were sitting there. Another simple example of residual heat is when a person walks across a floor while barefoot or wearing socks. This is shown in figure 3 . Thermal shadows in the form of a footprint appear in the places where a foot touched the floor as a result of conduction between the foot and the floor. Using an IR camera, students can continue to watch as the floor cools and the footprints disappear.

The real power of the camera in the classroom, however, is the fact that it can spark discussion and argumentation. All students have developed various conceptual models related to temperature and heat transfer. The IR camera will challenge 
some of these models and reinforce others. Through this continual self-validation of their own conceptions, the students will start to understand phenomena they observe and gain a deeper understanding of both the science content (temperature and heat transfer) and the inquiry skills (modeling and validation). For example, now that they understand the underlying concepts of temperature and heat, the students will be able to interpret processes involving conduction and other heat transfer phenomena.

\section{Research Rationale}

This research stems from ongoing collaboration with researchers at the Concord Consortium, an educational technology lab. Researchers at the Concord Consortium hypothesize that making computer simulations, in this case heat transfer simulations, an integral part of the design-based learning can enhance the learning efficacy in the classroom and stimulate students' curiosity towards heat transfer phenomena and related topics. Through an NSF-funded project "Engineering Energy Efficiency" [6], a 2D computational fluid dynamics program called Energy2D has been developed to teach heat transfer [7].

Energy $2 D$ is an interactive educational simulation tool that visualizes and explores the concepts of thermal energy, temperature distribution and heat transfer and may provide a straightforward method for presenting these concepts in the classroom [8]. Although Energy2D is limited to two-dimensional modeling, it is an accessible piece of software that provides the user with interactive visual representations of thermal energy, temperature distributions and heat and mass transfer [8] and is more than adequate in providing qualitative visualizations of these concepts. Based on this existing tool, the goal of the work presented here is to examine why an IR camera could be better for this task. Some of the drawbacks of Energy2D are the program's restriction to two-dimensions and models are presented are very idealized cases of heat transfer processes that are not fully representative of real-world situations.

Now that IR cameras are more accessible it is only natural that the tool is utilized to directly observe and demystify the mechanisms of heat transfer, which, until now, have been taught using temperature probes and simulations. This noncontact, interactive tool will give students the opportunity to engage with and make sense of ideas like heat and temperature. With a few materials and an IR camera any student can become a scientist and begin to explore the mechanisms of heat transfer. The combination of an easy to use software, such as Energy2D, with a direct observation capabilities of the IR camera, will allow students to dive deeper into the basic principles of what they observe. The following experiments are based on simulations from Energy2D and IR research conducted by Charles Xie and colleagues [6,7,9].

\section{Seeing Heat Transfer - Experiments for the Classroom}

A long wave $(7.5-13 \mu \mathrm{m}) \mathrm{Flir}^{\circledR}$ E30bx camera was used to examine the three modes of heat transfer: conduction, convection and radiation. The E30bx contains a $160 \times 120$ pixel detector and is accurate to within $0.1{ }^{\circ} \mathrm{C}$ [10], which is sufficient for the purposes of this research. To examine the heat transfer processes in real-time, the video feature of the camera was used with the Flir ${ }^{\circledR}$ IR Camera Player, a video capture software program provided by Flir $^{\circledR}$.

\subsection{Conduction}

There are a number of hands-on experiments demonstrating the effects of conduction such as melting ice cubes in one's hands or holding a metal spoon over a flame until the handle warms up. These experiments show the results of conduction, but they fail to show the underlying energy transfer, which is the cause of the ice cubes melting and the temperature increase in the spoon's handle. A simple experiment capturing heat flux in an aluminum bar was developed for students to "see" thermal conduction.

The setup, shown in figure 4, consists of a block of wood, a bar of aluminum, a coffee cup, a coffee cup warmer, two thermistors, a piece of fabric and a Styrofoam cooler which acts as an insulator to minimize energy losses due to convection. The cup warmer and empty coffee cup were placed in the cooler, and the block of wood was placed to the right of the cup in the cooler, so that both were at the same height. The aluminum bar, which was coated with flat white paint to ensure that its emissivity was close to the camera's setting (0.95), was placed on top of the cup and wood. One thermistor was secured to the side of the cooler about midway down to determine the surrounding temperature. The IR camera was positioned above and perpendicular to the setup in the cooler.

After turning on the cup warmer, used to preheat the cup to help maintain constant temperature of the liquid, and covering the top of the cooler and camera with fabric, to further minimize energy losses, the inside of the cooler was allowed the necessary time to reach an equilibrium temperature of $25{ }^{\circ} \mathrm{C}$. The water was then heated in an electric kettle and poured into the coffee cup via an aluminum foil funnel. The cup was filled to the very top so that the bottom side of the aluminum bar and hot water were in contact. 


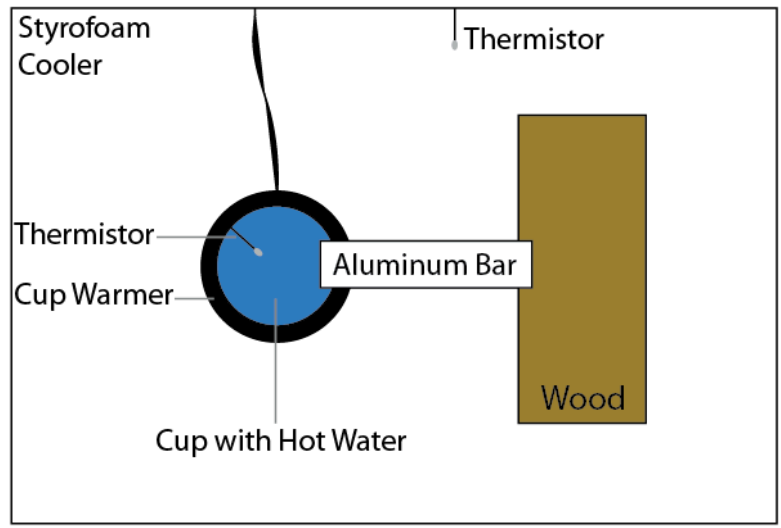

Figure 4. Experimental setup for conduction

Results of the experiment are shown in figure 5 below. The bar is initially at equilibrium with the surrounding air (first image) which begins to transport thermal energy from the hot water to the wood via conduction at the moment the bar and water make contact (second image). The water in the cup was $78{ }^{\circ} \mathrm{C}$ when conduction began, and three and a half minutes later the location of the bar marked by the crosshair reached $61{ }^{\circ} \mathrm{C}$ (last image). When the thermal energy conducted through the aluminum bar reaches the wood, the thermal energy begins to conduct to the wood. Notice that the rate at which thermal energy is conducted through the bar is much higher than the rate conducted to the wood, which reaches about $30{ }^{\circ} \mathrm{C}$ (indicated by the light blue color in the last image) in the three and a half minutes. The difference occurs because aluminum has a much higher thermal conductivity than wood.
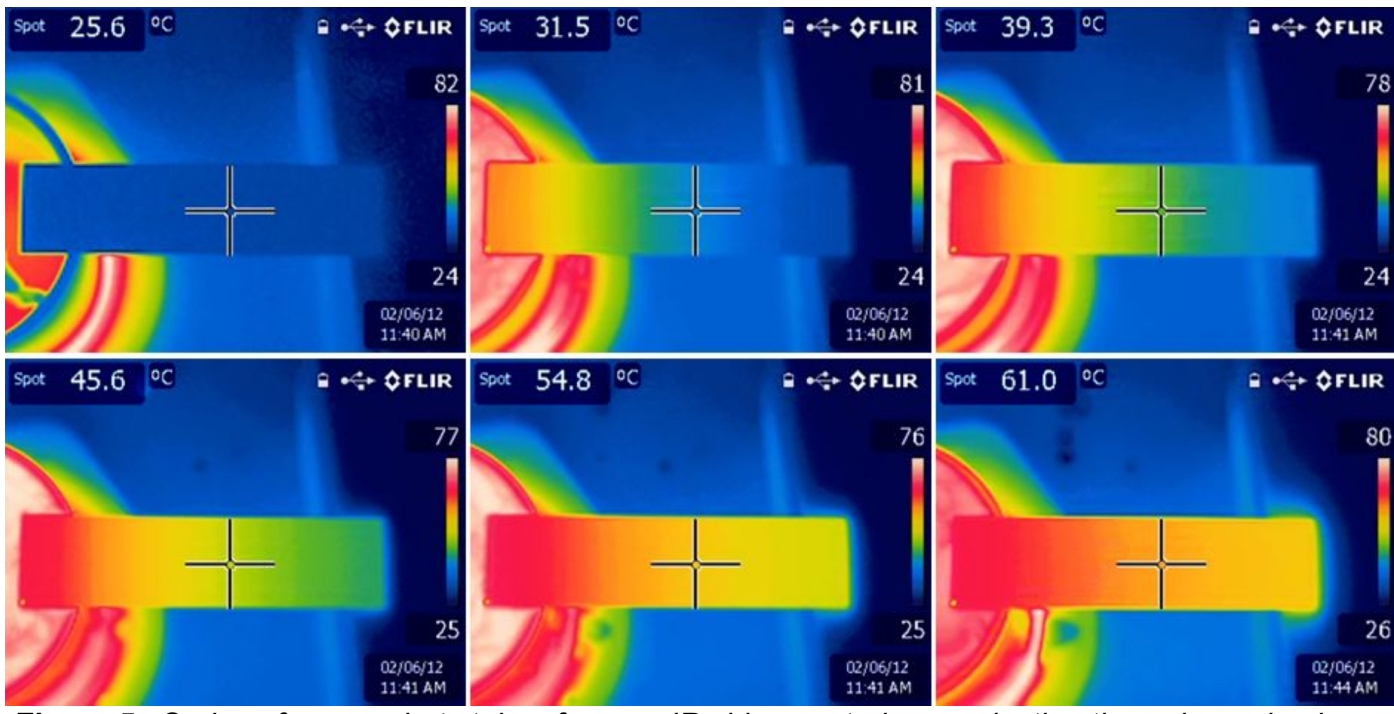

Figure 5. Series of screenshots taken from an IR video capturing conduction through an aluminum bar in real-time

As mentioned earlier, the cooler was used to minimize energy losses due to convection and turns out to be a very important element in the experimental setup. Initially, conduction through an aluminum bar was tested without any type of insulation present. The convective energy losses were so substantial that it took approximately four times longer for the temperature at the center of the bar to reach roughly $60{ }^{\circ} \mathrm{C}$. Although conduction was directly observed without using insulation, this setup is not ideal for use in the classroom due to the time needed to obtain clear results.

Figure 5 shows that this simple experiment successfully demonstrates the process of conduction. Using objects that are inexpensive and readily available along with an IR camera, students can now "see" what has been, up to this point, invisible. This experiment is not limited to the study of conduction but can also be used to investigate material properties such as specific heat, thermal conductivity and thermal resistance. Variations of this experiment include using bars made out of different materials, changing the temperature of the water, using different liquids, changing the length of the bar, etcetera. 


\subsection{Convection}

Air is invisible, not only to the human eye, but to the IR camera as well, which means convection, the transfer of energy through a fluid, in this case air, is also invisible to both the eye and IR camera. However, convection can be observed if the heat can be captured [9].

An experimental setup for seeing convection is shown in figure 6 and can be accomplished with a few simple steps. First, a rectangular channel, through which the warmed air will travel, is constructed using foam core and glue. The overhang is important to include on at least one end of the channel as it provides a way to suspend the channel above the hot plate and acts as a barrier to minimize convection from the areas of the hot plate that extend to either side of the channel. Two objects, in this case jars, of the same height were used to suspend the channel over a hot plate. With the channel at room temperature, shown in the first image of figure 7 , and the IR camera calibrated, the hot plate was turned on to $200{ }^{\circ} \mathrm{C}$.

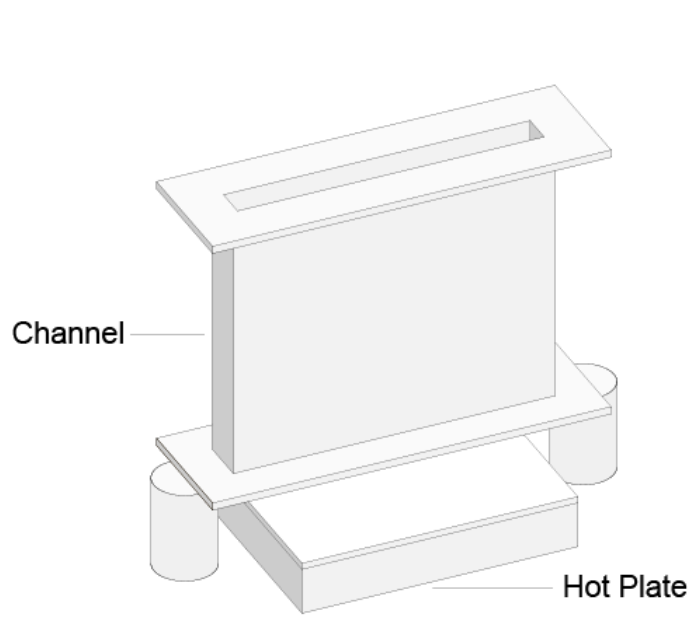

Figure 6. Experimental setup for conduction

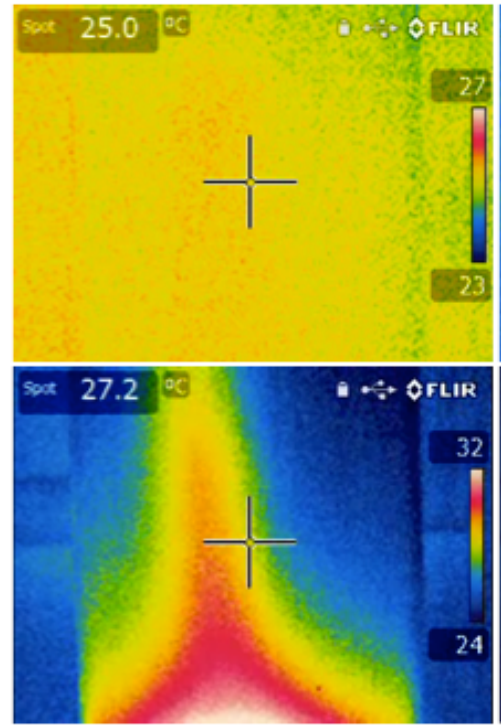

Figure 7. Series of screenshots taken from an IR video showing natural convection through an open channel
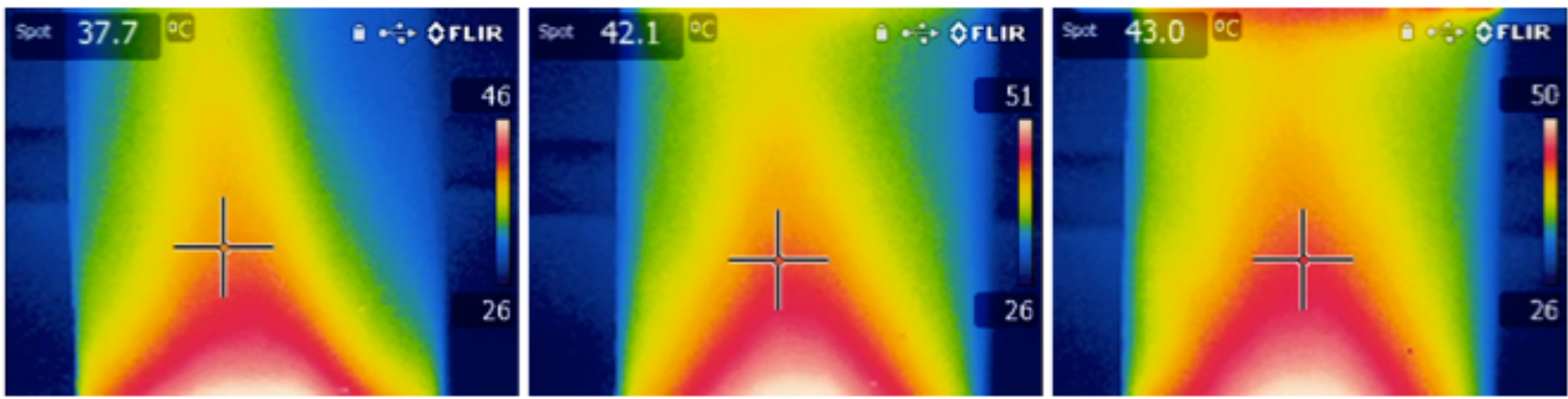

Figure 8. Series of screenshots taken from an IR video showing natural convection in a covered channel

Roughly thirty to forty-five seconds after turning the hot plate on (time will depend on the length of time it takes the hot plate to warm), a temperature gradient began to form on the channel, in image two of figure 7 . This gradient occurred as a result of the hot plate warming the air by transferring thermal energy to the cool surrounding air via convection. As the air right above the plate warms, its density decreases causing the warm air to rise. While moving up the channel thermal energy was transferred to the inner channel wall via convection, which then conducts to the outside of the wall revealing the convective pattern of the airflow. Notice that with the increase of time, the temperature gradient grows. This growth occurs because warm air that is rising begins to transfer energy to the walls at the opening of the channel, which causes a local temperature increase, image 2, and decreases the temperature difference between the air and the wall. Consequently, warm air following 
this decrease in temperature difference will transfer energy higher up the inside of the channel. These effects can be seen in the bottom images of figure 7 .

A cover can be placed on top of the channel as a representation of the effects a ceiling has in a room, see figure 8 . The same process described above is taking place, but the cover causes a build up of the warmed air at the top of the channel increasing the amount of thermal energy transferred at that location.

Additional experimentation is possible to explore convection in more depth. A few possible alterations include changing the temperature of the hot plate, adding small extrusions or fins along the channel wall and adjusting the dimensions of the channel. Further experimentation will provide opportunity for discussion regarding the effect of these alterations in relation to the original experiment and encourage students to create and explore adjustments of their own.

\subsection{Radiation}

Radiation is especially difficult for students to understand due to its abstract and unintuitive nature, but it is a phenomenon experienced numerous times a day. For instance, why does a person feel cold when facing a cold object? This experience is a result of the body emitting more radiation than the cold object. Invisible to the naked eye, students build a conceptual model of this process based on previous experiences and information obtained in the classroom. It only seems natural to use the IR camera to expose this heat transfer mechanism given that all objects emit radiation in the IR range [9].

This straightforward experiment reveals thermal radiation using a sheet of paper, which will act as the screen, a mason jar, hot and cold water, a ruler and tape. To set up the experiment, the sheet of paper should be fixed in an upright position so that the bottom of the paper is touching the table. Objects in the room, such as dry erase markers, can be secured to the two short edges of the paper using tape. The markers are stood upright to create the screen. Most important is that as much of the back of the screen as possible is unobstructed. For consistency a piece of tape was placed $10 \mathrm{~cm}$ from the back of the screen. The IR camera should be set up facing the side of the screen without the tape. Before beginning the experiment the camera parameters must be adjusted to ensure accurate temperature readings. See figure 9 for a schematic view of the experimental setup.
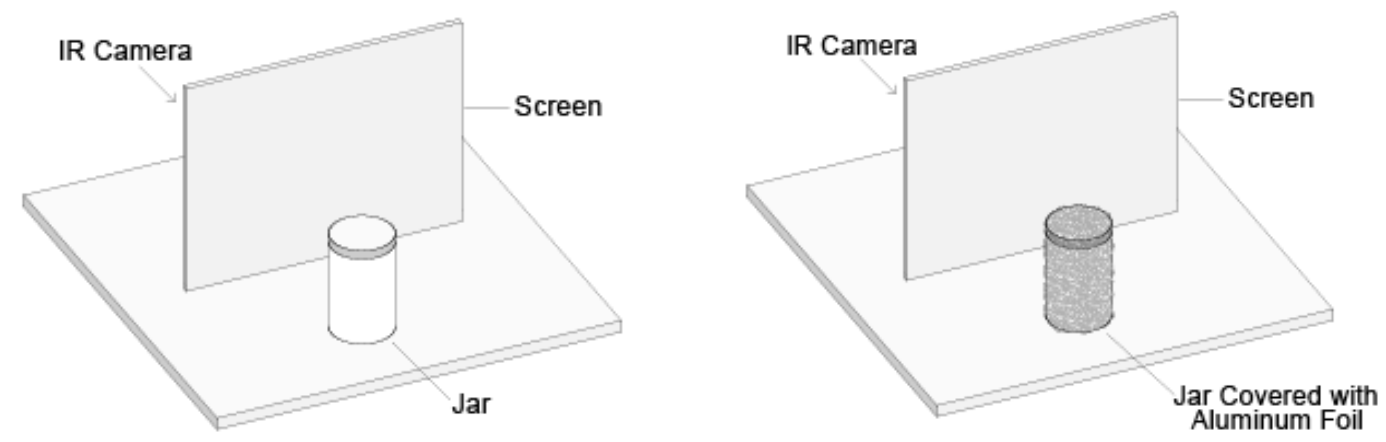

Figure 9. Experimental setup for radiation

The jar was placed on the tape then filled with hot water, $91{ }^{\circ} \mathrm{C}$, and sealed with the lid to reduce heat loss through evaporation and convection. To really observe the effects of radiative heat transfer, this experiment was repeated three more times, once using hot water and aluminum foil, ice water, at $0.1^{\circ} \mathrm{C}$, and ice water and aluminum foil.

Experimental results from the jar filled with hot water are shown in figure 10. The first image shows the screen at room temperature without the jar behind it. The second through the sixth images illustrate the development of a temperature gradient which increases with time as a result of energy transfer via radiation. Once the thermal radiation emitted from the jar reaches the screen, it is quickly conducted to the front of the screen because it is very thin. Note that the jar filled with hot water, was placed behind the screen so that the thermal radiation from the jar cannot reach the IR camera directly. 

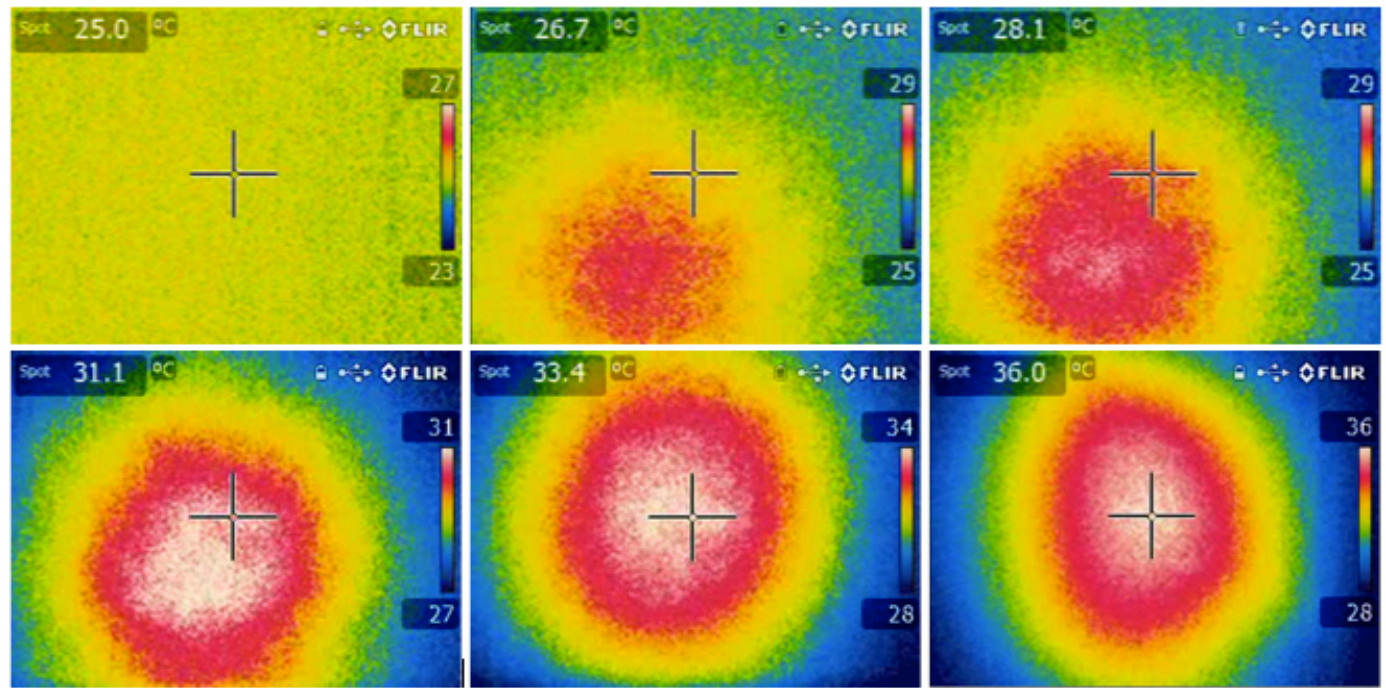

Figure 10. Series of screenshots taken from an IR video showing an increase in screen temperature due to radiation from a jar filled with hot water

For similar reasons, the cold water causes a temperature gradient that decreases with time. Figure 11 shows the screen temperature decreasing after the jar of cold water is introduced, again between the first and second images, and increasing to its initial temperature after the jar is removed, shown in the last three images. In these experiments very cold liquid was used to achieve clear results in a short amount of time.
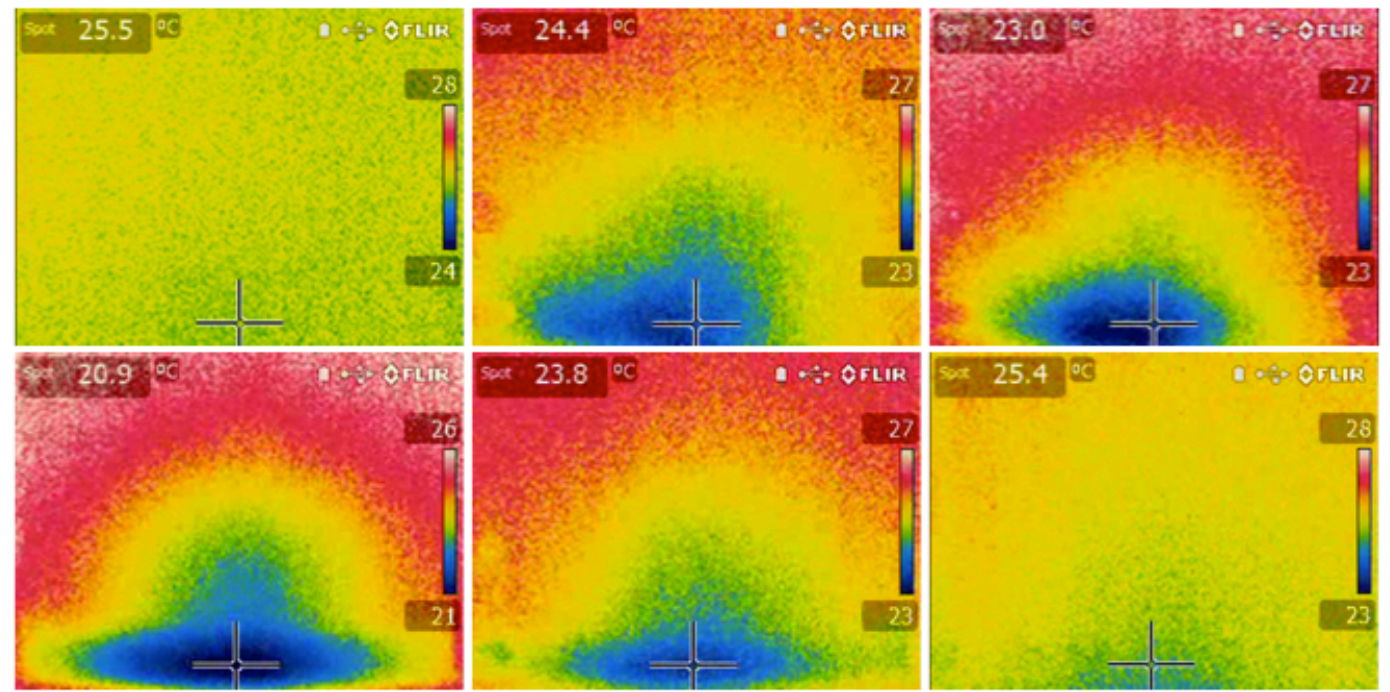

Figure 11. Series of screenshots taken from an IR video showing a decrease in screen temperature due to radiation from a jar filled with cold water

The effects of thermal radiation are further revealed completely wrapping the jar in aluminum foil and repeating the process previously described. The aluminum foil acts as a shield, preventing the transfer of energy via radiation. The covered jar is placed behind the screen, which is initially at rest in the first image of figures 12 and 13 . There is little to no increase or decrease in the temperature of the screen, from the hot or cold jar, respectively verifying that the aluminum foil was successful in preventing radiative heat transfer from the jar. A very small increase in temperature appears in figure 12 as a result of radiation from nearby objects being reflected off of the foil to the screen. 


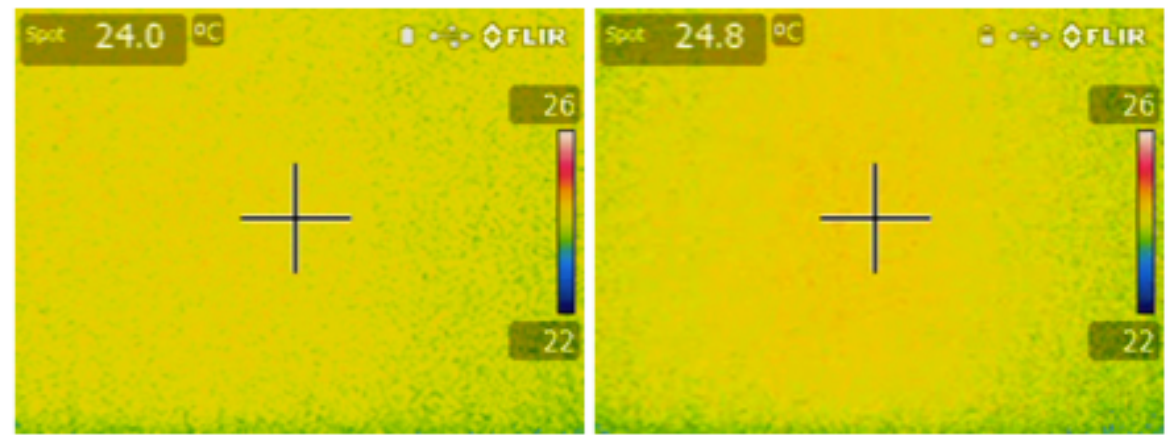

Figure 12. Series of screenshots taken from an IR video illustrating the effects of aluminum foil on radiative heat transfer (jar behind screen is filled with hot water)

The experiments involving ice water produced an intriguing "ufo" pattern on the screen as shown in figure 11. Although the cause of this result is not yet understood, one possible explanation is that a small amount of convection is reaching the screen due to the extremely low temperature of the water. The bottom part of the "ufo" pattern appears in figure 13even when the jar is covered with aluminum foil suggesting that the distance between the jar and the screen is not large enough and convection must be considered. When the temperature of the water increases, in the case when the jar is covered with foil, this pattern no longer exists as shown in the last image of figure 13.
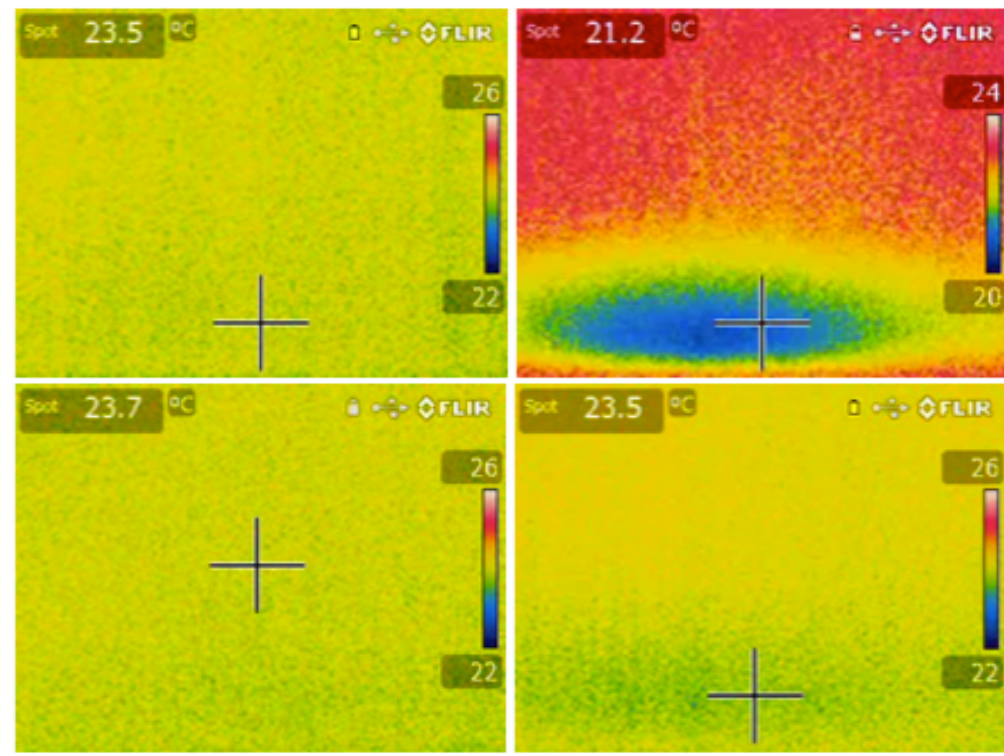

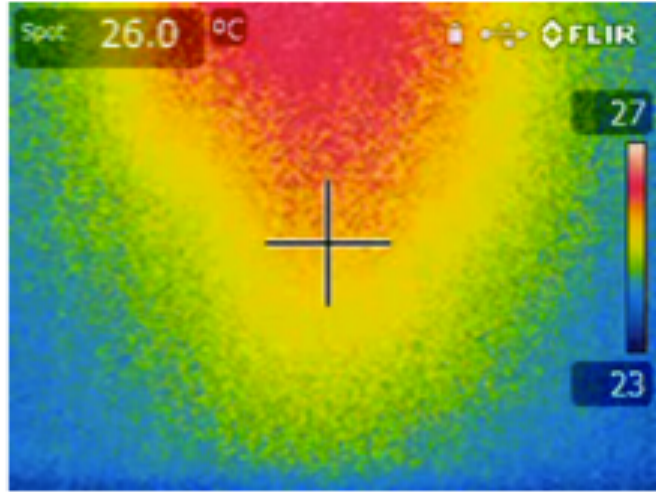

Figure 14. Screenshot from IR video showing radiative thermal energy transfer from a hand

Figure 13. Series of screenshots from an IR video illustrating the effects of aluminum foil on radiative heat transfer (water temperature $0.1{ }^{\circ} \mathrm{C}$ and $11.4{ }^{\circ} \mathrm{C}$ in the upper and lower two images, respectively)

There are many aspects of heat transfer at play here that can be studied and discussed in the classroom. Why is the change in temperature of the screen much larger with the jar filled with hot liquid? Why does the screen return to its initial temperature after the jar is removed faster in the experiment with the cold jar? What effect does the distance between the jar and the screen have on the temperature gradient shown on the screen? These are just a few discussion questions that will prompt students to think critically and investigate further. Additional experiments to explore some of the above questions include placing the jar at varying distances from the screen, changing the liquid temperature, using different liquids, placing various objects behind the screen, such as a hand shown in figure 14, etcetera.

\section{Energy2D and the IR Camera}

The drawbacks of the interactive simulation software, Energy2D, used to visualize heat and mass transfer were discussed earlier. Now that a conduction experiment, illustrating the transfer of energy through an aluminum bar in real time, has been 
developed, the limitations of the conduction simulation model can be explored. First, there are many assumptions and parameters affecting the model results in Energy2D that must be understood.

The conduction simulation model operates under the assumptions that both convection and radiation are not present, the thermal contact resistance between the aluminum bar and the wood is zero and the surrounding fluid, in this case air, is treated as a solid. Simply understanding the important role these assumptions play in the model is not enough. There are two parameters that have a significant affect on the simulated results. One is the time step length, which accounts for model accuracy. The default time step length maximizes simulation speed while maintaining accurate results. The second is the $z$ heat diffusivity, which accounts for energy losses to the atmosphere (where $z$ is the third dimension directed out of the screen).

Using the draw features in Energy2D, the experimental setup for conduction was reconstructed. After entering the material properties, leaving both the $z$ heat diffusivity and fluid conductivity set to their default values, zero, the model was run and the resulting simulation is shown in the first image of figure 15 . The center of the bar essentially reaches the temperature of the water, which is not realistic due to the substantial energy losses via convection to the surrounding fluid. To more accurately model the experiment presented earlier, the thermal conductivity of air was entered and the simulation was run multiple time to determine the necessary heat diffusion value was determined $(z=0.0181)$ producing results shown in the second image below. A model that closely matches thermal conduction captured with an IR camera was achieved by making the fluid conductivity almost zero.

The hands-on experiments developed in this paper are currently being used to examine the limitations of convection and radiation models provided in Energy2D.
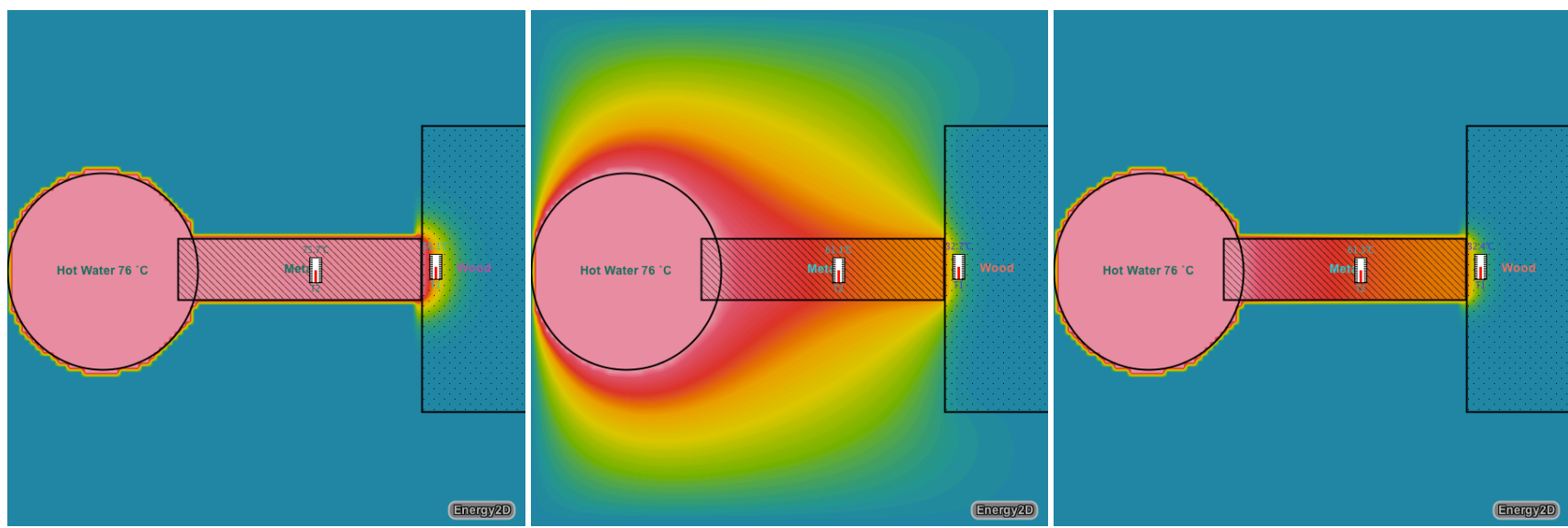

Figure 15. Screenshots of conduction simulations produced using Energy $2 \mathrm{D}-$ Image 1: Conductivity $=0 \mathrm{~W} / \mathrm{m} \cdot{ }^{\circ} \mathrm{C}, \mathrm{z}=0$; Image 2: Conductivity $=0.024 \mathrm{~W} / \mathrm{m} \cdot{ }^{\circ} \mathrm{C}, \mathrm{z}=0.0181$; Image 3 : Conductivity $=0.0001 \mathrm{~W} / \mathrm{m} \cdot{ }^{\circ} \mathrm{C}, \mathrm{z}=0.0181$

\section{Analysis of IR Images and Videos}

To fully engage students in thermal energy concepts, it is believed that having easy-to-use software that provides clear interpretations of IR images and videos they collect is important. As part of this research, an interactive tool for use in the classroom, which will enable students to record IR images and videos, showing them what they cannot see, will be developed. Additionally, software will be designed that allows them to easily analyze the images and videos they collect from the camera and plot different properties or different information over time. The combination of the IR camera with this software will give students the ability to share what they learn with a click of a button with classmates or friends, stimulating discussions or comments as observed in many other social network tools.

Currently, the only way to capture real-time video footage of heat and temperature on a simple IR camera such as the Flii ${ }^{\circledR}$ E30bx model is through difficult-to-find online software offered by Flir ${ }^{\circledR}$. The software is limited to use with Windows and must be downloaded and installed on each computer that will be used to record video footage. In order to make this technology more accessible and user-friendly, this research project will include the development of a simple and effective tool through LabVIEW $^{\circledR}$ that will not only allow the user to view and record IR video, but provide options for direct analysis of the collected data such as measurements, plotting, comparisons, with an option to publish results through a web portal. The user will be able to easily identify and manipulate temperature related characteristics such as isotherms and heat flux. A simplified 
version of what the user interface could look like is shown below in figure 16. Feedback from users will guide initial dynamic changes to the interface with the goal of obtaining the most intuitive and user-friendly interface possible.

The IR camera coupled with this software has the potential to open this new world of possibilities for the analysis on any IR images that any students feel worthwhile exploring. These tools and newly- developed skills will provide students with a new level of freedom to explore questions regarding, not only heat and temperature, but tangential components that may arise from discussion of these concepts while fostering learning and excitement towards science and technology.

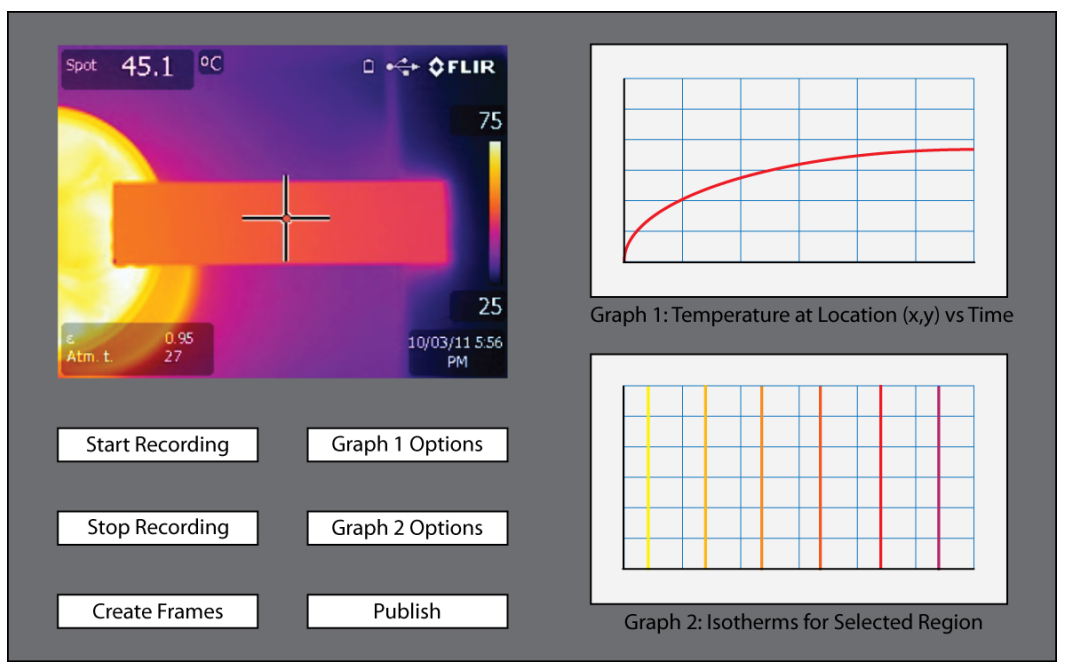

Figure 16. Simplified version of IR image and video data analysis

\section{Conclusions}

It is believed that the IR camera provides a unique opportunity to explore the mechanisms of heat transfer by visualizing of invisible phenomena in the natural world with which they already have some familiarity. Once the students can "see the unseen", their natural curiosity will lead to new thoughts, idea and more questions. This method of inquiry-based learning may act as a catalyst for scientific discussion in the classroom and lead to further analysis of unscripted questions bringing the learning experience to a completely new level where the learning is driven by inquisition rather than passive acceptance of pre-packaged explanations. By using this non-contact tool to study heat and temperature in real-time, students will begin to strengthen their problem-solving skills, data collection and analysis techniques, and expand their understanding of realworld issues such as energy efficiency while bolstering their curiosity and enthusiasm towards science and engineering.

As presented in this paper, it is possible to easily construct experiments highlighting different heat transfer phenomena such as conduction, convection and radiation. Until the cost of the IR camera drops enough for the product to be reachable by more people, including educators, Energy $2 D$ is a valuable interactive, easily accessible software program that allows students to visualize heat transfer concepts.

\section{REFERENCES}

[1] Erickson, G. L., \& Tiberghien, A., "Heat and temperature". In R. Driver, E. Guesne, \& A. Tiberghien (Eds.), Children's ideas in science, pp. 52-84, Philadelphia: Open University Press, 1985.

[2] N. C. on S. E. S. and Assessment and N. R. Council, National Science Education Standards, pp. 106, The National Academies Press, 1996.

[3] W. Hu, "Math That Moves: Schools Embrace the iPad". New York Times, 04-Jan-2011.

[4] F. Ion, "iPads in the Classroom are Changing the Face of Education". MacLife, 18-Apr-2012.

[5] http://www.military-dictionary.org/thermal_shadow

[6] "Engineering Energy Efficiency." http://energy.cocord.org

[7] "Energy2D." http://energy.concord.org/energy2d/

[8] Xie, C. "Interactive Heat Transfer Simulations for Everyone". The Physics Teacher, vol. 50, no. 4, pp. 237-240, 2012.

[9] C. Xie and E. Hazzard, "Infrared Imaging for Inquiry-Based Learning". The Physics Teacher, vol. 49, no. 6, pp. 368, 2011.

[10] “E30bx Specifications.” http://support.flir.com/DsDownload/Assets/49001-1701_en_50.pdf 